\title{
Prevalence of poor mental health among medical students in Nepal: a cross-sectional study
}

\author{
Arjab Adhikari ${ }^{1 * \dagger}$, Aman Dutta $^{1 \dagger}$, Supriya Sapkota ${ }^{1 \dagger}$, Abina Chapagain $^{1}$, Anurag Aryal ${ }^{2}$ and Amita Pradhan ${ }^{1}$
}

\begin{abstract}
Background: Poor mental health among medical students is widely acknowledged. Studies on mental health among medical students of Nepal are lacking. Therefore, we conducted a study to determine the prevalence of mental disorders.

Methods: A cross-sectional study was conducted among medical students at KIST Medical College and Teaching Hospital, Nepal from December 2016 to February 2017. Our survey instrument consisted of the Patient Health Questionnaire (PHQ) and questions about socio-demographic factors, smoking, marijuana use, suicidal ideation and thoughts of dropping out of medical school.

Results: The prevalence rates were $29.2 \%$ (95\% Cl, 24.4\% - 34.3\%) depression, $22.4 \%$ (95\% Cl, 18.0\% - 26.9\%) medium to highly severe somatic symptoms, $4.1 \%$ (95\% Cl, 2.0\% - 6.2\%) panic syndrome, 5.8\% (95\% Cl, 3.4\% - 8.3\%) other anxiety syndrome, 5\% (95\% Cl, 2.7\% - 7.3\%) binge eating disorder and 1.2\% (95\% Cl, 0.0\% - 2.3\%) bulimia nervosa. Sixteen students [4.7\% (95\% Cl, 2.4\% - 6.9\%)] seriously considered committing suicide while in medical school. Thirty-four students [9.9\% (95\% Cl, 6.8\% - 13.1\%)] considered dropping out of medical school within the past month. About 15\% (95\% Cl, 11.1\% - 18.6\%) of the students reported use of marijuana during medical school.

Conclusions: We found high prevalence of poor mental health among medical students of Nepal. Future studies are required to identify the factors associated with poor mental health.
\end{abstract}

Keywords: Mental health, Medical students, Nepal, Depression, Anxiety, Eating disorder, Somatic symptoms, Suicidal ideation, Dropping out, Marijuana

\section{Background}

The journey through medical school is a stressful and daunting task. High level of stress is seen in medical students [1]. Medical students at the first year of medical school have similar psychological morbidity compared to non-medical peers [2] and general population [3]. However, their mental health worsens as they course through medical school [2-4]. Despite having easy access to healthcare facilities, medical students are often reluctant to seek help for mental health concerns [5].

About one third of the medical students worldwide suffer from depression or depressive symptoms $[6,7]$.

\footnotetext{
*Correspondence: docarjab@gmail.com

${ }^{\dagger}$ Equal contributors

'KIST Medical College and Teaching Hospital, Lalitpur, Nepal

Full list of author information is available at the end of the article
}

Beside depression, anxiety and psychosomatic disorder [8] constitute an emerging mental health problem. Additionally, medical students are at high risk of developing eating disorders as well [9].

Poor mental health among medical students has been reported from various parts of Asia including India [10-12], Pakistan [13], Iran [14], Malaysia [15], China [16] and Saudi Arabia [4]. Poor mental health is found to be associated with serious thoughts of dropping out of medical school [17], substance abuse [18], burnout and suicidal ideation [19].

Studies on mental health of medical students in Nepal are lacking. Only few studies assessing depression [20, 21], anxiety [22] and suicidal ideation [23] were available on Pubmed, Google Scholar and Psycinfo database. Studies on other aspects of mental health including somatic 
symptoms and eating disorders were not available. Furthermore, the negative consequences of poor mental health including suicidal ideation, thoughts of dropping out of medical school and marijuana use are yet to be explored among Nepalese medical students. Thus, we conducted a survey to evaluate the following:

1. Prevalence of depression, somatic symptoms, anxiety syndromes and eating disorders

2. Prevalence of suicidal ideation, thoughts of dropping out of medical school and marijuana use

There are currently no programs in medical school curriculum of Nepal to screen for poor mental health or to cope with stress of being a medical student. Understanding of the mental health among medical students will encourage the development and integration of student wellness programs to prevent negative outcomes of poor mental health.

\section{Methods}

\section{Study design, setting and participants}

We conducted a cross-sectional survey at KIST Medical College and Teaching Hospital from December 2016 to February 2017. KIST Medical College and Teaching Hospital is affiliated to Tribhuvan University, Nepal. The medical school curriculum consists of basic science teaching in the first 2 years and clinical teaching in third, fourth and final year. The students in first 2 years have small element of clinical exposure. Final-year students were excluded from this study because of their unavailability as they were preparing for their board exams.

Our target was to include most of the students of first, second, third and fourth year. So, the questionnaire was distributed on a day with high number of attendees. Out of total 378 students, 370 were in attendance and received the questionnaire. Non-attendees and incomplete responses were excluded from the study. The study was conducted in the leisure time, in between the lectures. In a quiet classroom, after a short verbal presentation, a one-time, self-reported questionnaire was distributed. The cover letter consisted of an informed consent, which included a description of study and participants' rights to decline altogether or leave the questions unanswered. To preserve participants' anonymity, the questionnaire did not incorporate name, address or signature. Participants did not receive any incentives or financial compensation to participate in the study.

The total number of students in first-year and secondyear was higher than third- and fourth-year students (110 and 128 vs 69 and 71 respectively) because the number of students enrolled varies annually. Nepal Medical Council determines the number of students that can be enrolled annually by a medical school. At the time of data collection, first- and second-year students had completed their first month of the academic year. Third- and fourth-year students were in their final quarter of the academic year. The study was approved by Institutional Review Committee of KIST Medical College and Teaching Hospital.

\section{Survey tool}

Our survey instrument consisted of the Patient Health Questionnaire (PHQ) and questions about sociodemographic factors, smoking, marijuana use, suicidal ideation and thoughts of dropping out of medical school. Our survey instrument has been provided as Additional file 1 [see Questionnaire].

\section{PHQ}

PHQ is a validated, self-administered, three page questionnaire, which assesses depression, somatic symptoms, anxiety syndromes, alcohol abuse and eating disorders [24]. In our study, depression was assessed using PHQ - 9 modules. Somatic symptom severity was assessed using PHQ - 15 modules. PHQ algorithm was used to assess panic syndrome, other anxiety syndrome, binge eating disorder and bulimia nervosa. PHQ, PHQ - 9 modules, PHQ -15 modules and PHQ algorithm (instructions) are available at http://www.phqscreeners.com/.

\section{Depression}

PHQ requires students to rate each of the 9 depressive symptoms into 'not at all', 'several days', 'more than half the days' and 'nearly every day'. Using PHQ - 9 modules, scores of $0,1,2$ and 3 were assigned respectively. A cut - off score of 5, 10, 15 and 20 signifies mild, moderate, moderately severe and severe depression respectively. A score of $\geq 10$ has a sensitivity of $88 \%$ and specificity of $88 \%$ for major depression [25]. In this study, students scoring $\geq 10$ were considered having depression.

\section{Somatic symptoms}

PHQ requires students to rate each of the 13 somatic symptoms into 'not bothered at all', 'bothered a little' or 'bothered a lot'. Using PHQ -15 modules, scores of 0, 1 and 2 were assigned respectively. A cut-off score of 5,10 , and 15 signifies low, medium and high somatic symptoms severity respectively [26]. Students scoring $\geq 10$ were considered having medium to highly severe somatic symptoms.

\section{Panic syndrome and other anxiety syndrome}

PHQ can be used to diagnose both 'syndromes' and 'disorders'. However, diagnosis of 'disorders' from 'syndromes' requires further clinical interview. As our study was limited to self-administered questionnaire, our diagnosis was limited to 'syndromes' only. 


\section{Smoking}

Students were asked whether they had started smoking before or after joining medical school. Increment or decline in frequency of smoking was queried among students who started smoking before joining medical school.

\section{Marijuana use}

Students were asked to quantify the frequency of marijuana use before and during medical school into no use, 1-10 times, more than 10 times but less than each month, each month but less than each week, each week but not daily and daily use [27].

\section{Suicidal ideation and thoughts of dropping out}

To assess suicidal ideation, students were asked whether they had seriously considered committing suicide while in medical school. To assess thoughts of dropping out, students were asked whether they considered dropping out of medical school within the past month.

Positive screening for at least one syndrome identified by PHQ or having suicidal ideation was considered poor mental health in our study.

\section{Statistical analysis}

Chi-square analysis was used to test for association between study characteristics (gender, year in medical school) and mental health problems. Statistical analysis was done using IBM SPSS Statistics 23. $P$ value of $<0.05$ was considered significant for statistical test.

\section{Results}

Out of total 378 students, 370 were in attendance and received the questionnaire. Among 370 attendees, 343 $(92.7 \%)$ responses were complete. Incomplete responses were excluded from the study. The response rate was $92.59 \%, 94.4 \%, 94.11 \%$ and $88.40 \%$ in first-, second-, third- and fourth-year students respectively. The following results are based on 343 complete responses.

The prevalence rates were 29.2\% (95\% CI, 24.4\% $34.3 \%$ ) depression, $22.4 \%$ (95\% CI, $18.0 \%$ - 26.9\%) medium to highly severe somatic symptoms, $4.1 \%(95 \%$ CI, 2.0\% - 6.2\%) panic syndrome, 5.8\% (95\% CI, 3.4\% $8.3 \%)$ other anxiety syndrome, $5 \%$ (95\% CI, $2.7 \%-7.3 \%)$ binge eating disorder and $1.2 \%$ (95\% CI, $0.0 \%-2.3 \%$ ) bulimia nervosa. Table 1 shows the characteristics of study population.

\section{Depression}

The prevalence of depression was significantly higher in females compared to males $\left(35.1 \%\right.$ vs $23.4 \% ; X^{2}=5.672$, $P=0.017)$. A significantly higher percentage of preclinical students reported depression compared to clinical students (33\% vs $\left.22.4 \% ; X^{2}=4.344, P=0.037\right)$. Figure 1 shows the severity of depression.
Table 1 Characteristics of the study population

\begin{tabular}{ll}
\hline Study characteristics $(n=343)$ & \\
\hline Gender & $175(51 \%)$ \\
Male & $168(49 \%)$ \\
Female & \\
Year in medical school & $100(29.2 \%)$ \\
First & $118(34.4 \%)$ \\
Second & $64(18.7 \%)$ \\
Third & $61(17.8 \%)$ \\
Fourth & \\
Preclinical/Clinical & $218(63.6 \%)$ \\
Preclinical & $125(36.4 \%)$ \\
Clinical &
\end{tabular}

\section{Somatic symptoms and severity}

A significantly higher percentage of female students reported medium to highly severe somatic symptoms (30.4\% vs $\left.14.9 \% ; X^{2}=11.829, P=0.001\right)$. No significant difference was found between preclinical and clinical students. Figure 2 compares the frequency of somatic symptoms between male and female students. Among male students, headache was the most frequent complaint followed by pain in arms and legs. Menstrual cramps followed by headache were the most commonly reported complaints among female students.

\section{Anxiety syndromes}

The prevalence rates were panic syndrome, 5.8\% $(95 \%$ CI, $3.4 \%-8.3 \%)$ and other anxiety syndrome, 5\% (95\% CI, $2.7 \%-7.3 \%)$. No significant difference was found between male and female students as well as between preclinical and clinical students.

\section{Suicidal ideation}

Sixteen students (4.7\% [95\% CI, 2.4\% - 6.9\%]) seriously considered committing suicide while in medical school.

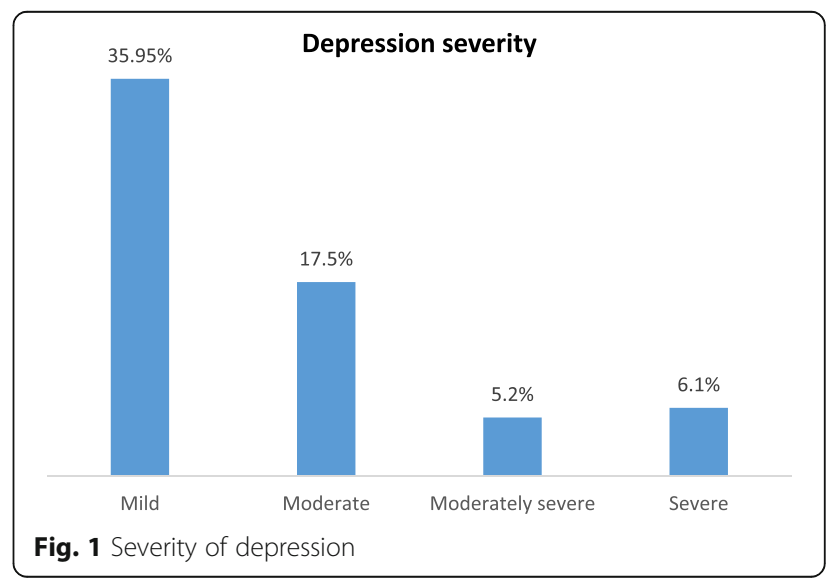



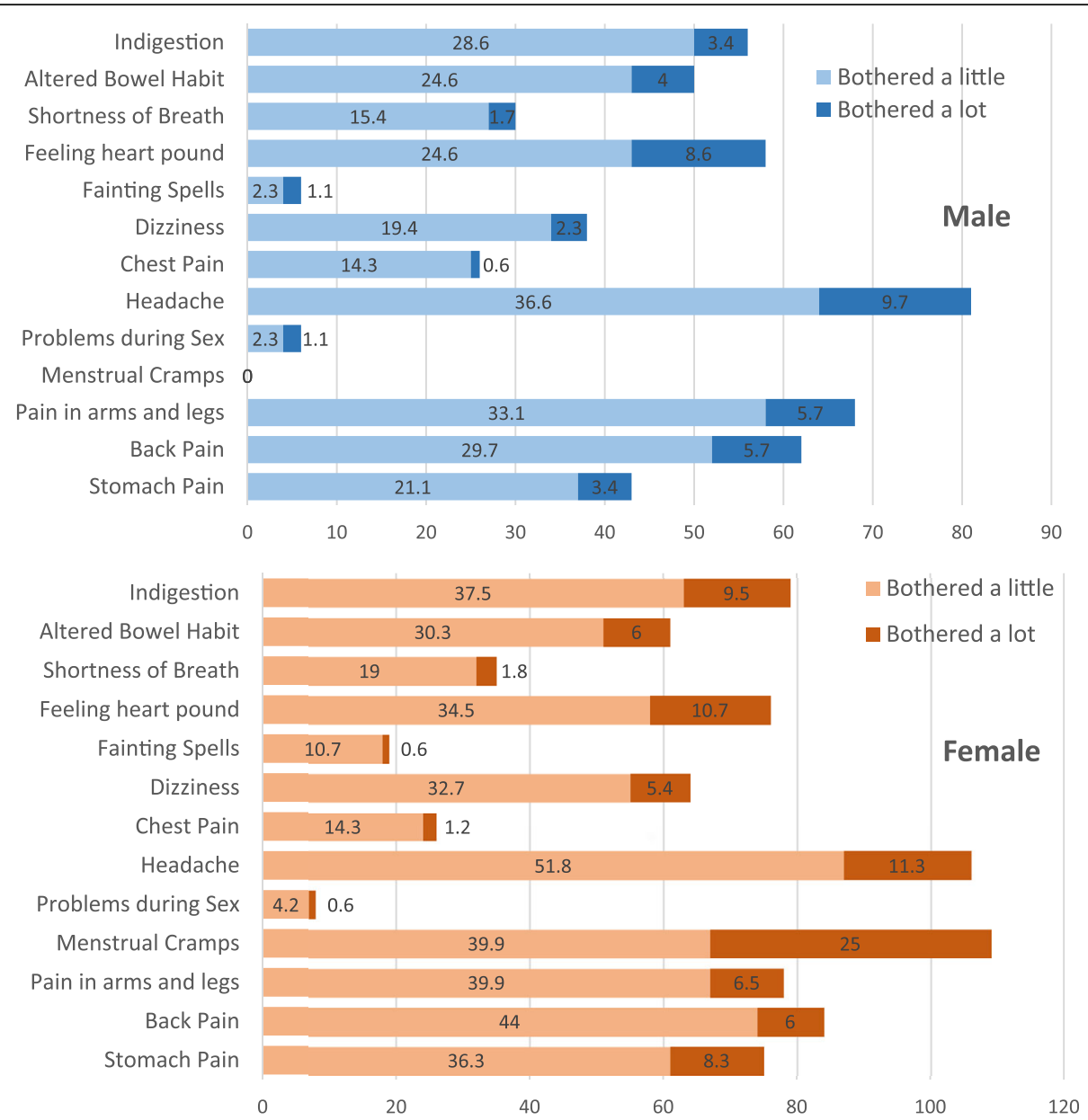

Fig. 2 Prevalence (percentage) of somatic symptoms among medical students

No significant difference was found between male and female students. Students in clinical years were more likely to report suicidal ideation compared to preclinical students ( $8 \%$ vs $\left.2.8 \% ; X^{2}=4.920, P=0.027\right)$. A significantly higher percentage of depressed students reported suicidal ideation compared to students without depression $\left(12 \%\right.$ vs $\left.1.6 \% ; \mathrm{X}^{2}=17.078, P=0.000\right)$.

\section{Thoughts of dropping out}

Thirty-four students (9.9\% [95\% CI, 6.8\% - 13.1\%]) considered dropping out of medical school within the past month. No significant difference was found between male and female students as well as between preclinical and clinical students. Students with depression were more likely to have thoughts of dropping out compared to students without depression (19\% vs $6.2 \% ; \mathrm{X}^{2}=$ 13.053, $P=0.000$ ).

\section{Eating disorder}

Binge eating disorder was present in 5\% (95\% CI, 2.7\% 7.3\%) of the students. About 10\% of the students with depression were also positive for binge eating disorder. This relation was statistically significant $\left(X^{2}=7.997\right.$, $P=0.005)$. No significant difference was found between male and female students.

\section{Smoking}

The prevalence of smoking among medical students was $14.3 \%$ (95\% CI, 10.6\% - 18.0\%). The prevalence of smoking was significantly higher in males compared to females $\left(26.3 \%\right.$ vs $\left.1.8 \% ; X^{2}=42.018, P=0.000\right)$. Majority of students $(69 \%)$ started smoking before joining medical school. Among them, 47\% reported an increase in frequency of smoking after joining medical school whereas $53 \%$ of them reported decrease in smoking frequency.

\section{Marijuana use}

About 15\% [95\% CI, 2.7\% - 7.3\%] of the students reported use of marijuana during medical school. Table 2 shows the frequency of marijuana use before and during medical school. Before joining medical school 3.49\% of students had used marijuana 10 times or more whereas 
Table 2 Frequency of marijuana use before and during medical school

\begin{tabular}{lll}
\hline Frequency & $\begin{array}{l}\text { Before medical } \\
\text { school }\end{array}$ & $\begin{array}{l}\text { During medical } \\
\text { school }\end{array}$ \\
\hline No use & 314 & 292 \\
$1-10$ times & 17 & 31 \\
$\begin{array}{l}\text { More than } 10 \text { times but less } \\
\text { than each month }\end{array}$ & 5 & 2 \\
$\begin{array}{l}\text { Each month but less than } \\
\text { each week }\end{array}$ & 1 & 5 \\
$\begin{array}{l}\text { Each week but not daily } \\
\text { Daily use }\end{array}$ & 3 & 8 \\
\hline
\end{tabular}

$5.83 \%$ of the students used marijuana 10 times or more during medical school. Marijuana use during medical school was significantly higher in male students than in female students $\left(24.6 \%\right.$ vs $\left.4.8 \% ; X^{2}=26.573, P=0.000\right)$. Students with depression were no more likely than students without depression to report marijuana use (15\% vs $\left.14.8 \% ; X^{2}=0.002, P=0.965\right)$. Similarly, we found no significant association between other mental disorders (medium to highly severe somatic symptoms, panic syndrome and other anxiety syndrome) and marijuana use.

\section{Discussion}

The aim of our study was to determine the prevalence of various mental disorders among medical students of Nepal. In Nepal, the prevalence of depression among general population of age group 18-25 years is $2.5 \%$ [28]. In our study, we found the prevalence to be $29.2 \%$ which is higher than the general population. However, the prevalence in general population was determined using hospital anxiety and depression scale compared to PHQ used in our study. Our finding is comparable with recent metaanalysis studies $27.2 \%$ [7], 28\% [6] as well as previous studies from Nepal 29.8\% [20], 29.9\% [22].

The current study found that $22.4 \%$ of students had medium to highly severe somatic symptoms. This percentage is high compared to medical students in Germany, where the prevalence is about 15\% [29]. Our finding supports the idea that psychosomatic disorder is an emerging mental health problem among medical students [8].

We found the prevalence of panic syndrome and other anxiety syndrome to be $4.1 \%$ and $5.8 \%$ respectively. Using same questionnaire, the prevalence of panic syndrome and other anxiety syndrome in German medical students was $4.4 \%$ and $1.9 \%$ respectively [29]. Using Depression Anxiety and Stress Scale, Kunwar et al. [22] found $41.1 \%$ of the students in two medical colleges of Nepal had anxiety disorder. This difference may be due to different study instrument used.

In this study, the prevalence of eating disorders was $5 \%$ binge eating and $1.2 \%$ bulimia nervosa. There was a significant positive correlation between depression and binge eating disorder. These results match those observed in earlier studies $[9,30]$.

According to a recent meta-analysis by Rotenstein et al., the pooled prevalence of suicidal ideation among medical students is $11.1 \%$ [7]. The results of this study show a prevalence of $4.7 \%$. When compared to the same meta-analysis, an interesting finding was that the prevalence of depression was higher in our study but the prevalence of suicidal ideation was lower. This finding is difficult to explain. Differences in socio-cultural aspects may be responsible. A prospective study showed that suicidal ideation during medical school predicted postgraduate suicidal ideation [19]. Such thoughts persists into practice as well [31]. So, it is necessary to effectively deal with such thoughts in time.

To our knowledge, this is the first study to report thoughts about dropping out and marijuana use among Nepalese medical students. Our result was similar to a multi-institutional study in US [17], which reported $11 \%$ of the students had thoughts of dropping out. Marijuana is second to alcohol as the most common substance to be abused by medical students [32]. It is more commonly used to 'feel good' and 'have a good time' [32] than as a coping strategy to stress [33].The reason for marijuana use among Nepalese medical students should be addressed in future studies. We hope that our findings will provide future researchers with comparative data.

\section{Gender}

Females were more likely to have medium to highly severe somatic symptoms and depression. Previous studies report varying relationship between female gender and depression. Some studies [10, 13] show higher prevalence in female students while other studies $[11,12,14]$ show no difference. High prevalence of depression seen in female medical students has been linked to personality traits [34], gender inequity and associated stigma [35].

\section{Year in medical school}

Prevalence of depression was significantly higher in preclinical students. Similarly, studies from Pakistan [13] and India [11] report higher prevalence of depression in preclinical students. Longitudinal studies $[3,36]$ done in American medical students have shown high prevalence of depression in second year of medical school. The observed high rate of depression in second-year students in our study may be due to academic burnout. Students must pass the second year-university exams in first two attempts. Otherwise, they will have to face a delay of 1 year before attempting again. Similarly, human cadaver dissection which is done in preclinical year is stressful for many students [37]. Depression in first-year students may be due to stress of having to face a new challenging 
environment [38]. In Nepal, most students enroll into medical college directly from high school, compared to US students who enter medical school with a bachelor degree [39]. This may cause an abrupt increase in academic burden, leading to stress among first-year students.

\section{Recommendations}

Future studies are required to identify and explore the factors associated with poor mental health among Nepalese medical students. There are currently no programs to identify and help students with their mental health and substance abuse problems. Various solutions to reduce stress like creating a nurturing learning environment, identifying and assisting students, teaching skills for stress management and promoting self-awareness have been proposed [38]. Integration of such programs as a part of medical education with special focus on preclinical students and female students may improve mental health among students.

\section{Limitations}

Samples in this study were from a single medical school. As the study was conducted in the classroom, students were in close seating arrangement which may have affected students' privacy leading to under representation of results of smoking and marijuana use.

\section{Conclusions}

We found high prevalence of poor mental health among medical students of Nepal. Future studies are required to identify the factors associated with poor mental health.

\section{Additional file}

Additional file 1: Survey instrument. Contains questionnaire of the study. (PDF 831 kb)

\section{Abbreviations}

Cl: Confidence interval; DSM - IV: Diagnostic and Statistical Manual of mental disorders - IV; PHQ: Patient Health Questionnaire

\section{Acknowledgements}

We would like to thank the students for their participation. We would also like to thank Mr. Nabin Ban of KIST Medical College and Teaching Hospital for helping us to co-ordinate with the students.

\section{Funding}

No funding was received from any sources.

\section{Availability of data and materials}

The raw datasets of this study can be made available from the corresponding author on reasonable request.

\section{Authors' contributions}

Arjab Adhikari (AA) came up with the idea and designed the study with the help of AD and SS. Acquisition of data was done by SS and AC with help from $A A$ and $A D$. Anurag Aryal (AAR) entered the data into SPSS with the help from SS. AP analyzed and interpreted the data with the help from AD and AA. SS and AC conducted the focus group discussion. All the authors contributed in preparation and submission of manuscript. All authors read and approved the final manuscript.

\section{Ethics approval and consent to participate}

The study was approved by Institutional Review Committee of KIST Medical College and Teaching Hospital. Informed written consent was taken from participants.

Consent for publication

Not applicable.

\section{Competing interests}

The authors declare that they have no competing interests.

\section{Publisher's Note}

Springer Nature remains neutral with regard to jurisdictional claims in published maps and institutional affiliations.

\section{Author details}

${ }^{1}$ KIST Medical College and Teaching Hospital, Lalitpur, Nepal. ${ }^{2}$ Kathmandu Medical College and Teaching Hospital, Kathmandu, Nepal.

Received: 22 April 2017 Accepted: 21 November 2017

Published online: 28 November 2017

\section{References}

1. MacLean L, Booza J, Balon R. The impact of medical school on student mental health. Acad Psychiatry. 2016;40:89-91.

2. Carson AJ, Dias S, Johnston A, McLoughlin MA, O'Connor M, Robinson BL, et al. Mental health in medical students. A case control study using the 60 item general health questionnaire. Scott. Med. J. 2000;45:115-6.

3. Rosal MC, Ockene IS, Ockene JK, Barrett SV, Ma Y, Hebert JR. A longitudinal study of students' depression at one medical school. Acad. Med. J. Assoc. Am. Med. Coll. 1997:72:542-6.

4. Yusoff MSB, Mat Pa MN, Esa AR, Abdul Rahim AF. Mental health of medical students before and during medical education: a prospective study. J Taibah Univ Med Sci. 2013:8:86-92.

5. Tijia J, Givens JL, Shea JA. Factors associated with undertreatment of medical student depression. J Am Coll Health J ACH. 2005;53:219-24.

6. Puthran R, Zhang MWB, Tam WW, Ho RC. Prevalence of depression amongst medical students: a meta-analysis. Med Educ. 2016;50:456-68.

7. Rotenstein LS, Ramos MA, Torre M, Segal JB, Peluso MJ, Guille C, et al. Prevalence of depression, depressive symptoms, and suicidal ideation among medical students: a systematic review and meta-analysis. JAMA. 2016:316:2214-36.

8. Chinawa JM, Nwokocha ARC, Manyike PC, Chinawa AT, Aniwada EC, Ndukuba AC. Psychosomatic problems among medical students: a myth or reality? Int J Ment Health Syst [Internet] 2016 [cited 2017 15;10. Available from: http://www.ncbi.nlm.nih.gov/pmc/articles/PMC5123430/.

9. Memon AA, Adil SE-R, Siddiqui EU, Naeem SS, Ali SA, Mehmood K. Eating disorders in medical students of Karachi, Pakistan-a cross-sectional study. BMC Res. Notes. 2012;5:84.

10. Iqbal S, Gupta S, Venkatarao E. Stress, anxiety \& depression among medical undergraduate students \& their socio-demographic correlates. Indian J Med Res. 2015;141:354-7.

11. Kumar GS, Jain A, Hegde S. Prevalence of depression and its associated factors using Beck depression inventory among students of a medical college in Karnataka. Indian J Psychiatry. 2012;54:223-6.

12. Vankar JR, Prabhakaran A, Sharma H. Depression and stigma in medical students at a private medical college. Indian J Psychol Med. 2014:36:246-54.

13. Jadoon NA, Yaqoob R, Raza A, Shehzad MA, Zeshan SC. Anxiety and depression among medical students: a cross-sectional study. JPMA J Pak Med Assoc. 2010;60:699-702.

14. Farahangiz S, Mohebpour F, Salehi A. Assessment of mental health among Iranian medical students: a cross-sectional study. Int J Health Sci. 2016;10:49-55

15. Tan ST, Sherina MS, Rampal L, Normala I. Prevalence and predictors of suicidality among medical students in a public university. Med J Malaysia. 2015;70:1-5.

16. Sobowale K, Zhou AN, Fan J, Liu N, Sherer R. Depression and suicidal ideation in medical students in China: a call for wellness curricula. Int J Med Educ. 2014;5:31-6. 
17. Dyrbye LN, Thomas MR, Power DV, Durning S, Moutier C, Massie FS, et al. Burnout and serious thoughts of dropping out of medical school: a multi-institutional study. Acad Med J Assoc Am Med Coll. 2010;85:94-102.

18. Khan MS, Mahmood S, Badshah A, Ali SU, Jamal Y. Prevalence of depression, anxiety and their associated factors among medical students in Karachi, Pakistan. J Pak Med Assoc. 2006:56:583-6.

19. Tyssen R, Vaglum P, Grønvold NT, Ekeberg O. Suicidal ideation among medical students and young physicians: a nationwide and prospective study of prevalence and predictors. J Affect Disord. 2001;64:69-79.

20. Basnet B, Jaiswal M, Adhikari B, Shyangwa PM. Depression among undergraduate medical students. Kathmandu Univ Med J KUMJ. 2012;10:56-9.

21. Abramova T, Ogunji A, Taiwo OO. Assessment of depression and stress among medical students from three different countries: Nepal, Ukraine and Russia: A Cross-Sectional Survey. [cited 2017 Apr 10]; Available from: http://www.academia.edu/19590582/Assessment_of_Depression_and_ Stress_among_Medical_Students_from_Three_Different_Countries_Nepal_ Ukraine_and_Russia_A_Cross-Sectional_Survey.

22. Kunwar D, Risal A, Koirala S. Study of depression, anxiety and stress among the medical students in two medical colleges of nepal. Kathmandu Univ Med J. 2016;53:22-6.

23. Menezes RG, Subba SH, Sathian B, Kharoshah MA, Senthilkumaran S, Pant $S$, et al. Suicidal ideation among students of a medical college in western Nepal: a cross-sectional study. Legal Med. 2012;14:183-7.

24. Spitzer RL, Kroenke K, Williams JB. Validation and utility of a self-report version of PRIME-MD: the PHQ primary care study. Primary care evaluation of mental disorders. Patient health questionnaire. JAMA. 1999:282:1737-44.

25. Kroenke K, Spitzer RL, Williams JB. The PHQ-9: validity of a brief depression severity measure. J Gen Intern Med. 2001;16:606-13.

26. Kroenke K, Spitzer RL, Williams JBW. The PHQ-15: validity of a new measure for evaluating the severity of somatic symptoms. Psychosom Med. 2002;64:258-66.

27. Schwartz RH, Lewis DC, Hoffmann NG, Kyriazi N. Cocaine and marijuana use by medical students before and during medical school. Arch Intern Med. 1990;150:883-6.

28. Risal A, Manandhar K, Linde M, Steiner TJ, Holen A. Anxiety and depression in Nepal: prevalence, comorbidity and associations. BMC Psychiatry. 2016;16:102

29. Wege N, Muth T, Li J, Angerer P. Mental health among currently enrolled medical students in Germany. Public Health. 2016;132:92-100.

30. Manaf NA, Saravanan C, Zuhrah B. The prevalence and inter-relationship of negative body image perception, depression and susceptibility to eating disorders among female medical undergraduate students. J Clin Diagn Res. 2016;10:VC01-4.

31. Hem E, GrŁnvold NT, Aasland OG, Ekeberg O. The prevalence of suicidal ideation and suicidal attempts among Norwegian physicians. Results from a cross-sectional survey of a nationwide sample. Eur. Psychiatry J. Assoc. Eur. Psychiatr. 2000;15:183-9.

32. Baldwin DC, Hughes PH, Conard SE, Storr CL, Sheehan DV. Substance use among senior medical students: a survey of 23 medical schools. JAMA. 1991;265:2074-8.

33. Sreeramareddy CT, Shankar PR, Binu V, Mukhopadhyay C, Ray B, Menezes RG. Psychological morbidity, sources of stress and coping strategies among undergraduate medical students of Nepal. BMC Med Educ. 2007;7:26.

34. Hojat M, Glaser K, Xu G, Veloski JJ, Christian EB. Gender comparisons of medical students' psychosocial profiles. Med Educ. 1999;33:342-9.

35. Bleakley A. Gender matters in medical education. Med Educ. 2013;47:59-70.

36. Clark DC, Zeldow PB. Vicissitudes of depressed mood during four years of medical school. JAMA. 1988;260:2521-8.

37. Guthrie EA, Black D, Shaw CM, Hamilton J, Creed FH, Tomenson B. Embarking upon a medical career: psychological morbidity in first year medical students. Med Educ. 1995:29:337-41.

38. Dyrbye LN, Thomas MR, Shanafelt TD. Medical student distress: causes, consequences, and proposed solutions. Mayo Clin Proc. 2005;80:1613-22.

39. Dyrbye LN, Thomas MR, Shanafelt TD. Systematic review of depression, anxiety, and other indicators of psychological distress among U.S. and Canadian medical students. Acad. Med. J. Assoc. Am Med Coll. 2006;81:354-73.

\section{Submit your next manuscript to BioMed Central and we will help you at every step:}

- We accept pre-submission inquiries

- Our selector tool helps you to find the most relevant journal

- We provide round the clock customer support

- Convenient online submission

- Thorough peer review

- Inclusion in PubMed and all major indexing services

- Maximum visibility for your research

Submit your manuscript at www.biomedcentral.com/submit
Biomed Central 